\title{
Utilização do ANSYS como laboratório virtual no ensino remoto
}

DOI: https://doi.org/10.35168/2176-896X.UTP.Tuiuti.2020.Vol7.N62.pp46-63

Alexandre Guilherme de Lara

Professor da Universidade Tuiuti do Paraná. Doutorando na área de Mecânica dos Sólidos no Programa de Pós-graduação em Engenharia Mecânica e de Materiais da UTFPR. Mestre na área de Engenharia Biomédica da UTFPR (PPGEB).

E-mail: alexandre.lara1@utp.br

Angela Helena Zatti

Professora da Universidade Tuiuti do Paraná - UTP. Mestrado em Comunicação e Linguagens - UTP.

E-mail: angela.zatti@utp.br

Tatyane Moura

Professora da Universidade Tuiuti do Paraná - UTP. Mestrado em Educação pela Universidade Tuiuti do Paraná.

E-mail: tatyane.moura@utp.br Orcid: https://orcid.org/0000-0002-7544-6889

Revista Tuiuti: Ciência e Cultura, v.7 n.62. p. 46-63, Curitiba, 2021 


\section{Utilização do ANSYS como laboratório virtual no ensino remoto}

\section{Resumo}

O presente trabalho se propõe apresentar o software ANSYS como laboratório virtual no ensino remoto em engenharia. Com o avanço dos recursos tecnológicos e computacionais, tais como programas e equipamentos computacionais, verifica-se uma oportunidade de utilização de tais recursos no ensino remoto da engenharia. Em razão da pandemia de COVID 19, o ensino presencial foi temporariamente suspenso por razões de saúde, deixando uma lacuna de demonstrações experimentais, comuns no ensino da Engenharia. Neste trabalho apresentaremos a utilização do ANSYS como ferramenta no ensino remoto da engenharia, na forma de um laboratório virtual em demonstrações de elementos estruturais. Buscou-se demonstrar, a título de exemplo, a deformação elástica de uma viga, normalmente observada em ensaio mecânico de flexão.

Palavras-chave: Ensino remoto. Engenharia. Laboratório virtual. ANSYS. 


\section{ANSYS as virtual lab in remote teaching}

\section{Abstract}

The present work presents ANSYS software as a virtual laboratory in remote engineering teaching. Considering the advances in technological and computational resources, such as software and equipment, an opportunity take place in apply these resources in remote engineering education. Due to the COVID 19 pandemic, classroom teaching was temporarily suspended, leaving a gap in experimental demonstrations. Such demonstrations are common in engineering education. This work presents the use of ANSYS as a tool in remote teaching as a Virtual Lab. One example has been used to show the behavior of an elastic beam under constant stress, and the correspondent deformation of the specimen.

Keywords: Remote education. Engineering. Virtual lab. ANSYS. 


\section{Utilização do ANSYS como laboratório virtual no ensino remoto}

\section{Introdução}

Em razão da pandemia de COVID 19, a reitoria da Universidade Tuiuti do Paraná, amparada pelas portarias 343 e 345 do Ministério da Educação, e por resoluções dos órgãos Colegiados da Universidade, determinou a migração das aulas presenciais para o regime letivo remoto. O período letivo remoto teve início em meados de março de 2020, e se estendeu ao longo do ano letivo (UNIVERSIDADE TUIUTI, 2020). No sentido de assegurar o ensino aos alunos do curso de Engenharia Mecânica, a coordenação, juntamente com o corpo docente do curso, buscou a adoção de ferramentas computacionais que pudessem ser empregadas em substituição às demonstrações experimentais presenciais.

A ferramenta computacional adotada na área estrutural foi o software ANSYS, cujo desenvolvedor permitiu, ao longo de 2020, a aquisição da licença estudantil de forma gratuita. A versão estudantil permite a utilização de praticamente todos os recursos do software, tendo como principal restrição a limitação do número de elementos nodais.

Para fins do presente trabalho, delimitou-se a aplicação da ferramenta em análise estrutural para um exemplo envolvendo a deformação elástica de viga. Com este exemplo, buscou-se verificar a possibilidade de observação dos mesmos fenômenos que seriam observados em uma análise experimental presencial. Em seguida, analisaram-se as semelhanças e diferenças da simulação no ambiente virtual em relação ao ambiente experimental.

A hipótese proposta é que o software ANSYS, ferramenta computacional de método de elementos finitos, pode ser utilizado de forma satisfatória como sendo um laboratório virtual. Para essa análise, uma lista de características observáveis dos fenômenos será utilizada, de forma a comparar o que se observa no laboratório experimental e no virtual. 


\section{Utilização do ANSYS como laboratório virtual no ensino remoto}

\section{Fundamentação}

Segundo Piaget (apud TAFNER, 2021), a aprendizagem decorre dos processos de assimilação e acomodação, sendo o primeiro um processo cognitivo de captar as várias informações expostas no meio e integrar, assimilando as informações em estruturas cognitivas já existentes. O processo de acomodação, por conseguinte, consiste uma modificação dos meios de assimilação sob a influência de situações externas (meio), permitindo desenvolver as capacidades cognitivas, em maior ou menor grau.

Segundo Demo (2007) a teoria deve ser combinada à prática, pois dessa forma a teoria se confronta com a prática, renovando-se, e a prática sempre se volta à teoria, refazendo-se. Neste contexto, o Projeto Teórico-Prático busca tornar os alunos agentes ativos no processo de aprendizagem, desenvolvendo o questionamento reconstrutivo.

A associação da prática com a teoria é uma questão axiomática contida no Projeto Pedagógico do curso de Bacharelado em Engenharia Mecânica da Universidade Tuiuti do Paraná (ENGENHARIA MECÂNICA TUIUTI, 2021). As Diretrizes Curriculares Nacionais (DCNs) das Engenharias, normatizadas na resolução no 02 da Câmara de Educação Superior do Ministério da Educação (BRASIL, 2019), institui no seu artigo sexto a obrigatoriedade de atividades de laboratório para o desenvolvimento de competências gerais e específicas. Também estimula a articulação de maneira simultânea entre teoria e prática, bem como o uso de metodologias de aprendizagem ativa.

A ferramenta computacional utilizada neste estudo é o pacote de software ANSYS, que emprega o Método de Elementos Finitos (MEF) na simulação de diversos fenômenos. Segundo Jacob e Ted (2009) MEF é uma aproximação numérica com a qual equações diferenciais parciais ligadas a determinados fenômenos físicos podem ser resolvidas de modo aproximado. Do ponto de vista da engenharia, o MEF é um método para resolver problemas de engenharia, tais como análise 


\section{Utilização do ANSYS como laboratório virtual no ensino remoto}

de tensões, transferência de calor, escoamento de fluidos e eletromagnetismo, por simulações de computador. O pacote ANSYS permite diversas destas análises nas áreas de: fluidodinâmica, estrutural, multifísica e eletromagnética.

\section{Cálculo de tensão em viga sujeira a flexão}

Segundo Richard e Keith (2016), as equações para tensões normais em vigas retas se baseiam nas seguintes hipóteses:

- O material é isotrópico e homogêneo;

- O material obedece à lei de Hooke;

- Inicialmente, a viga é reta, com seção transversal constante ao longo de seu comprimento;

- A viga está submetida à flexão pura. Isso significa que a força de cisalhamento é zero e que não existem cargas axiais de torção;

- A viga possui um eixo de simetria no plano de flexão;

- As proporções da viga são tais que ela falharia por flexão e não por esmagamento, enrugamento ou flambagem;

- Seções transversais planas da viga permanecem planas durante a flexão.

Com estas hipóteses é possível realizar a modelagem matemática da viga.

A tensão de flexão varia linearmente com a distância a partir do eixo neutro, y, e é dada por:

$$
\sigma_{x}=-\frac{M y}{I}
$$




\section{Utilização do ANSYS como laboratório virtual no ensino remoto}

em que: $\sigma_{\mathrm{x}}$ é a tensão [MPa], o M é o momento fletor [Nm], o y é a distância da linha neutra ao ponto considerado $[\mathrm{m}]$ e o I é o momento de inércia $\left[\mathrm{mm}^{4}\right]$.

A tensão de cisalhamento máxima, por sua vez, é:

$$
\tau_{\text {máx }}=\frac{3 V}{2 A}
$$

As tensões principais, por sua vez, são estimadas em um estado plano de tensão por:

$$
\sigma_{\text {mín,máx }}=\frac{\sigma_{x}^{2}+\sigma_{Y}^{2}}{2} \pm \sqrt{\left(\frac{\sigma_{x}^{2}-\sigma_{Y}^{2}}{2}\right)+\tau_{X Y}^{2}}
$$

Segundo a norma de dimensionamento de estruturas metálicas 8800, e citando a norma ABNT NBR 7007, são adotados os seguintes valores para a propriedade mecânica dos aços: módulo de elasticidade $\mathrm{E}_{\mathrm{a}}=200.000 \mathrm{MPa}$, coeficiente de Poisson $v_{\mathrm{a}}=0,3$, resistência ao escoamento $\mathrm{f}_{\mathrm{y}}=250$ $\mathrm{MPa}$ e resistência à ruptura do aço à tração $\mathrm{f}_{\mathrm{u}}=400 \mathrm{MPa}$. (ASSOCIAÇÃO BRASILEIRA DE NORMAS TÉCNICAS, 2008).

O cálculo da flecha é apresentado na equação 4 (GUIA DE ENGENHARIA, 2021):

$$
\delta=\frac{5 q L^{4}}{384 E I}
$$




\section{Utilização do ANSYS como laboratório virtual no ensino remoto}

\section{Ensaio de tração e flexão}

De acordo com Callister (2020) as propriedades mecânicas dos materiais são verificadas por meio de experimentos de laboratório que reproduzem as condições de serviço de determinado material. Dentre os ensaios mecânicos, um dos mais comuns é o ensaio de tração, no qual uma amostra é tracionada e deformada até a ruptura. O corpo de prova apresenta uma forma de "osso de cachorro", para que a deformação fique confinada à região mais estreita do corpo de prova.

A deformação pode ser elástica ou plástica. A deformação elástica não é permanente, o que significa que, quando a carga aplicada é liberada, a peça retorna à sua forma original. Nesta região é válida a Lei de Hooke, descrita pela equação 5 como:

$$
\sigma=\mathrm{E} \varepsilon
$$

e a constante de proporcionalidade $E$ (com unidades de GPa ou psi) é o módulo de elasticidade, ou módulo de Young (CALLISTER, 2020). Já a deformação plástica ocorre após o limite de proporcionalidade, ou limite de escoamento, e apresentará um comportamento distinto para materiais frágeis e dúcteis. A deformação plástica é caracterizada por ser permanente e cumulativa. A figura 01 apresenta um esquema do comportamento mecânico de um corpo de prova.

Segundo Souza (1982), a determinação das propriedades mecânicas de um material metálico é obtida por meio de diversos ensaios, geralmente destrutivos, porque promovem a ruptura ou inutilização do material. Na categoria dos destrutivos, o autor destaca os ensaios de tração, dobramento, flexão, torção, fadiga e impacto, sendo que a reprodutividade e facilidade de execução do ensaio de tração o tornam o mais importante destes ensaios mecânicos. A figura 1 ilustra um ensaio mecânico de flexão. 


\section{Utilização do ANSYS como laboratório virtual no ensino remoto}

O ensaio de tração é utilizado para determinação das propriedades mecânicas de determinado material. Contudo é importante ressaltar que o ensaio de tração convencional difere do ensaio de tração real, na medida em que o primeiro não considera a alteração de geometria sofrida pelo corpo de prova ao longo do ensaio, o que afetaria o cálculo de tensão. Isto significa que o ensaio de tração convencional está sujeito a erros (SOUZA, 1982).

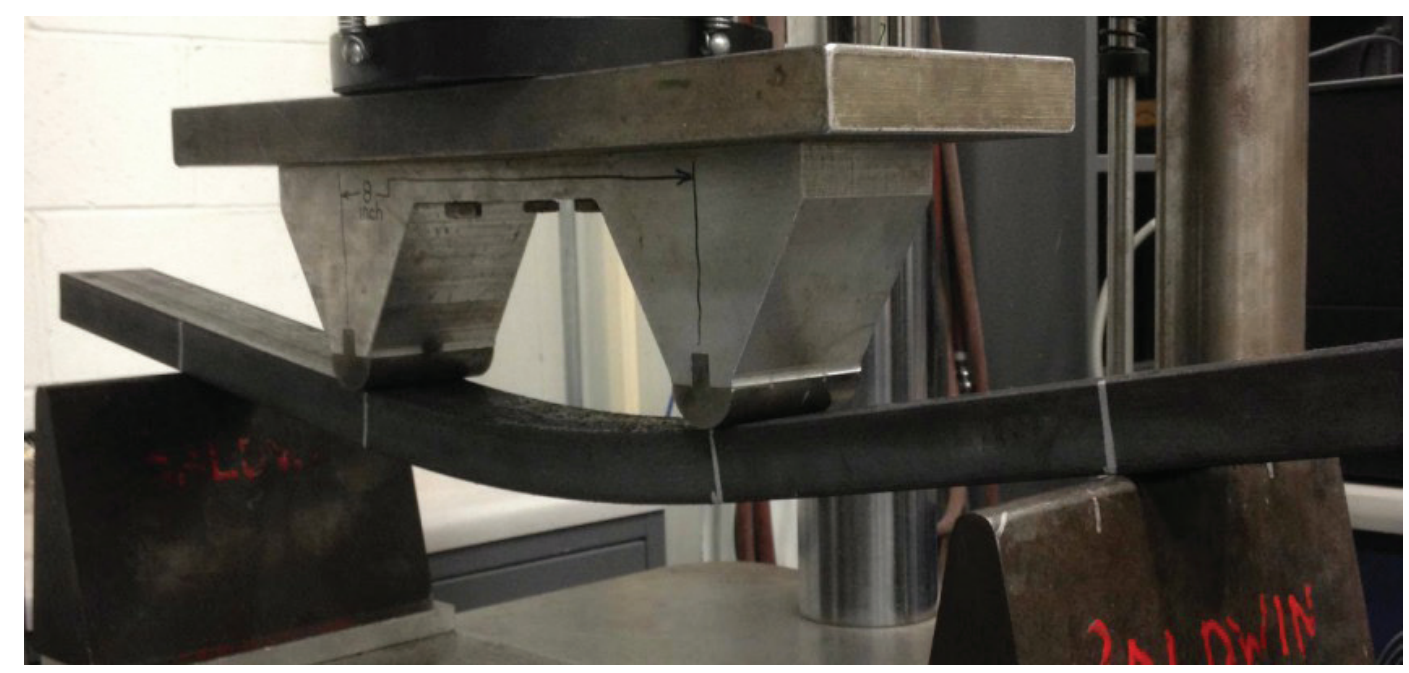

Figura 01 - Ensaio mecânico de flexão

Fonte: Callister, 2020 (Adaptado)

\section{Metodologia}

O presente trabalho se propõe analisar a utilização do que denominamos laboratório virtual em substituição ao laboratório experimental físico, em razão da necessidade de adequação de aula 


\section{Utilização do ANSYS como laboratório virtual no ensino remoto}

do regime presencial para o regime remoto. Segundo Lakatos (2021), neste tipo de artigo busca-se uma análise e cada elemento constitutivo do assunto e sua relação com o todo. Neste sentido, o principal aspecto é o de verificar os elementos que podem ser observados em ensaios mecânicos de laboratório de flexão e de tração.

Para isto serão relacionados os principais aspectos primários e secundários de cada um dos ensaios e, em seguida, fazer uma análise do assunto e a relação entre as partes.

Como aspecto primário dos ensaios mecânicos de tração e flexão, para determinação das propriedades físicas, entende-se:

1. Determinação da tensão;

2. Determinação do deslocamento;

3. Relação entre tensão e deformação;

4. Verificação dos conceitos de elasticidade.

Como aspectos secundários dos ensaios mecânicos de tração e flexão, para determinação das propriedades físicas, entende-se:
A. Determinação do gráfico: tensão vs deformação;
B. Possibilidade de alteração de parâmetros de ensaio;
C. Verificação de fenômenos secundários, como o associado ao coeficiente de Poisson;
D. Alongamento, estricção, e limite de ruptura;
E. Distinção entre o ensaio de tração convencional e o ensaio de tração real. 


\section{Utilização do ANSYS como laboratório virtual no ensino remoto}

\section{Resultados}

Analisaremos o emprego do laboratório virtual em uma deflexão elástica em viga de perfil em I (VS). O exemplo utilizado é o proposto pela ESSS (2020). A figura 02 ilustra o elemento estrutural simulado numericamente. A viga apresenta configuração biapoiada, com 8,0 metros de vão, seção transversal em perfil VS com dimensões de 400,0 × 78,0 mm com 400,0 x 200,0 x 19,0 × 7,0mm, em aço estrutural ASTM 36, considerando um carregamento distribuído com $250 \mathrm{KN}$ sobre a viga.

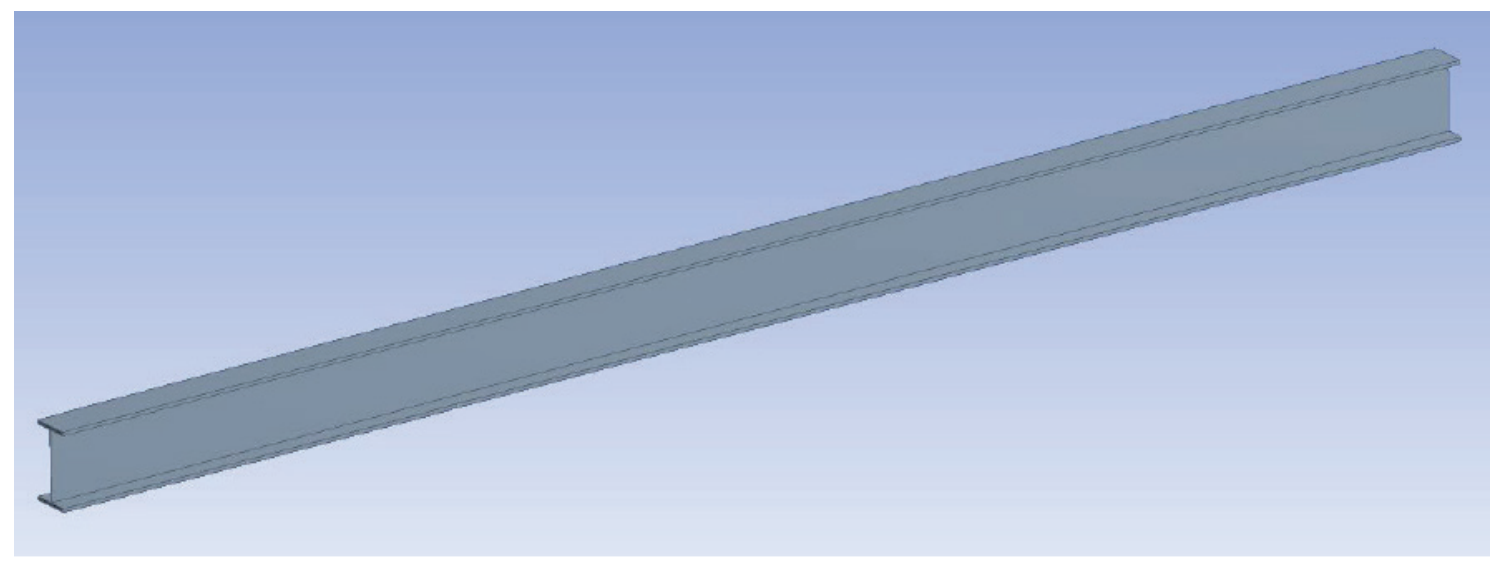

Figura 2 - Viga ensaiada

Fonte: ESSS, 2000 (Adaptado)

Primeiramente buscou-se uma solução analítica do problema para, em seguida, buscar a solução numérica por meio dos métodos de elementos finitos.

Sabendo que a equação de momento para o caso em tela é deduzida como sendo $\mathrm{M}(\mathrm{x})=$ $-15625 \mathrm{x} 2+125000 \mathrm{x}$, verificamos que o momento fletor máximo é de $\mathrm{M}(\mathrm{x})=250.000 \mathrm{~N} \cdot \mathrm{mm}$. 


\section{Utilização do ANSYS como laboratório virtual no ensino remoto}

Considerando para o perfil de uma viga VS 400 x 78, cujo momento de inércia em relação ao eixo $\mathrm{x}$ é de $\mathrm{Ix}-\mathrm{x}=3,0094 \mathrm{x} 108 \mathrm{~mm} 4$, de área $\mathrm{A}=9.880,0 \mathrm{~mm} 2$, e os seus valores de carregamento e dimensionais apresentados anteriormente, podemos calcular a tensão de flexão com a equação 6, conforme a seguir ilustrado:

$$
\sigma=-\frac{M y}{I}=-\frac{(250.000 \mathrm{~N} \cdot \mathrm{mm})(219 \mathrm{~mm})}{3,094 \cdot 10^{8}}=-181,92 \mathrm{MPa}
$$

O valor de cisalhamento pode ser calculado como sendo:

$$
\tau=\frac{3 V}{2 A}=\frac{3(250.000 N)}{2(9.880)}=37,95 \mathrm{MPa}
$$

Sabendo que o carregamento distribuído é de $250 \mathrm{KN}$, e que a viga tem $8.000,0 \mathrm{~mm}$ de comprimento, é possível afirmar que o carregamento é de q=31,25N/mm. O cálculo de deflexão da viga segue a equação 4:

$$
\delta=\frac{5 q L^{4}}{384 E I}=\frac{5(31,25)(8.000)^{4}}{384(200.000)\left(3,0094 \times 10^{8}\right)}=27,69 \mathrm{~mm}
$$

Em seguida solucionamos pelo método de elementos finitos no ANSYS, conforme pode ser observado pelas figuras 3 e 4 : 


\section{Utilização do ANSYS como laboratório virtual no ensino remoto}

\begin{tabular}{|c|c|c|c|c|c|}
\hline \multicolumn{6}{|c|}{ Definition } \\
\hline Type & Total Deformation & Equivalent (von-Mises) Stress & Normal Stress & Shear Stress & Normal Stress \\
\hline By & \multicolumn{5}{|c|}{ Time } \\
\hline Display Time & \multicolumn{5}{|c|}{ Last } \\
\hline Calculate Time History & \multicolumn{5}{|c|}{ Yes } \\
\hline \multicolumn{6}{|l|}{ Identifier } \\
\hline Suppressed & \multicolumn{5}{|c|}{ No } \\
\hline Orientation & & & XAxis & $X Z$ Component & Z Axis \\
\hline Coordinate System & & & \multicolumn{3}{|c|}{ Global Coordinate System } \\
\hline \multicolumn{6}{|c|}{ Results } \\
\hline Minimum & $0,33469 \mathrm{~mm}$ & $0,77737 \mathrm{MPa}$ & $-148,93 \mathrm{MPa}$ & $-49,79 \mathrm{MPa}$ & $-169,48 \mathrm{MPa}$ \\
\hline Maximum & $24,712 \mathrm{~mm}$ & $244,28 \mathrm{MPa}$ & $150,03 \mathrm{MPa}$ & $49,79 \mathrm{MPa}$ & $3,5993 \mathrm{MPa}$ \\
\hline Average & $15,924 \mathrm{~mm}$ & $87,478 \mathrm{MPa}$ & $-0,25052 \mathrm{MPa}$ & $-1,9225 \mathrm{e}-008 \mathrm{MPa}$ & $-2,1174 \mathrm{MPa}$ \\
\hline Minimum Occurs On & Midsurface - SolidMidsurface2 & \multicolumn{2}{|c|}{ Midsurface - SolidMMidsurface 3} & \multicolumn{2}{|c|}{ Midsurface - SolidMMidsurface1 } \\
\hline Maximum Occurs On & Midsurface - SolidMMidsurface3 & \multicolumn{2}{|c|}{ Midsurface - SolidMMidsurface2 } & \multicolumn{2}{|c|}{ Midsurface - SolidMMidsurface1 } \\
\hline
\end{tabular}

Figura 3 - Resultados ANSYS

Fonte: Autoria própria

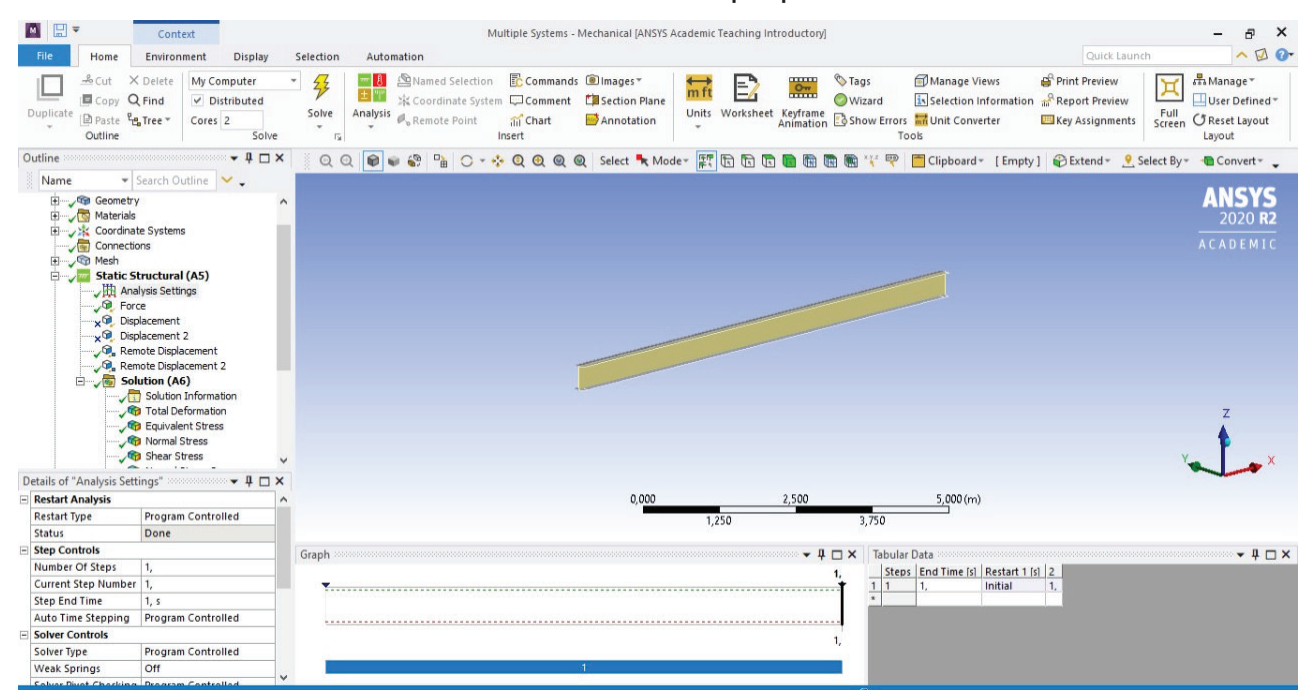

Figura 4 - Viga perfil I submetida à flexão.

Fonte: Autoria própria. 


\section{Utilização do ANSYS como laboratório virtual no ensino remoto}

A figura 5 ilustra a viga submetida a uma deformação plástica por flexão, cuja tensão máxima observada foi de $\sigma=150 \mathrm{MPa}$, e a figura 5 a deflexão de .

A figura 6 ilustra a resposta numérica para a tensão de cisalhamento, com valor de tensão $\tau=49,79 \mathrm{MPa}$.
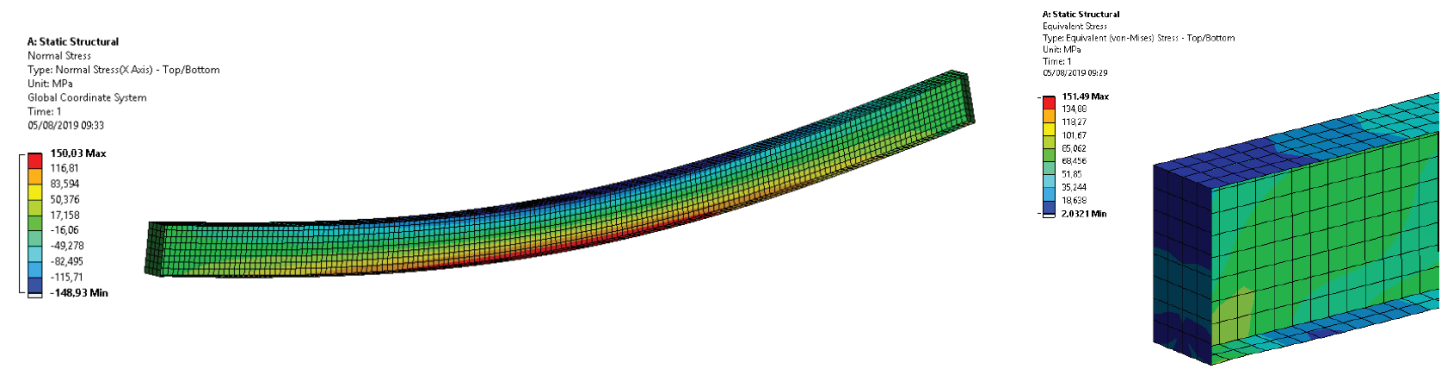

Figura 5 - Viga perfil I submetida a flexão, com valor de 150,03 MPa de tensão.

Fonte: ESSS, 2000 (Adaptado)

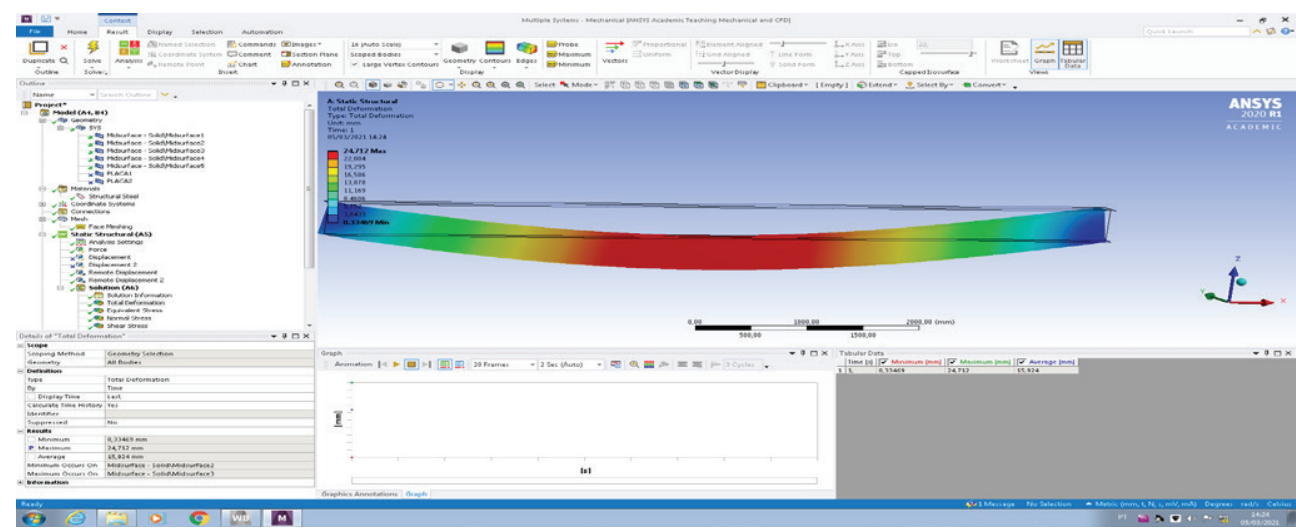

Figura 6 - Deflexão da viga em relação a sua condição não deformada ( =24,7 mm). Fonte: Autoria própria. 


\section{Utilização do ANSYS como laboratório virtual no ensino remoto}

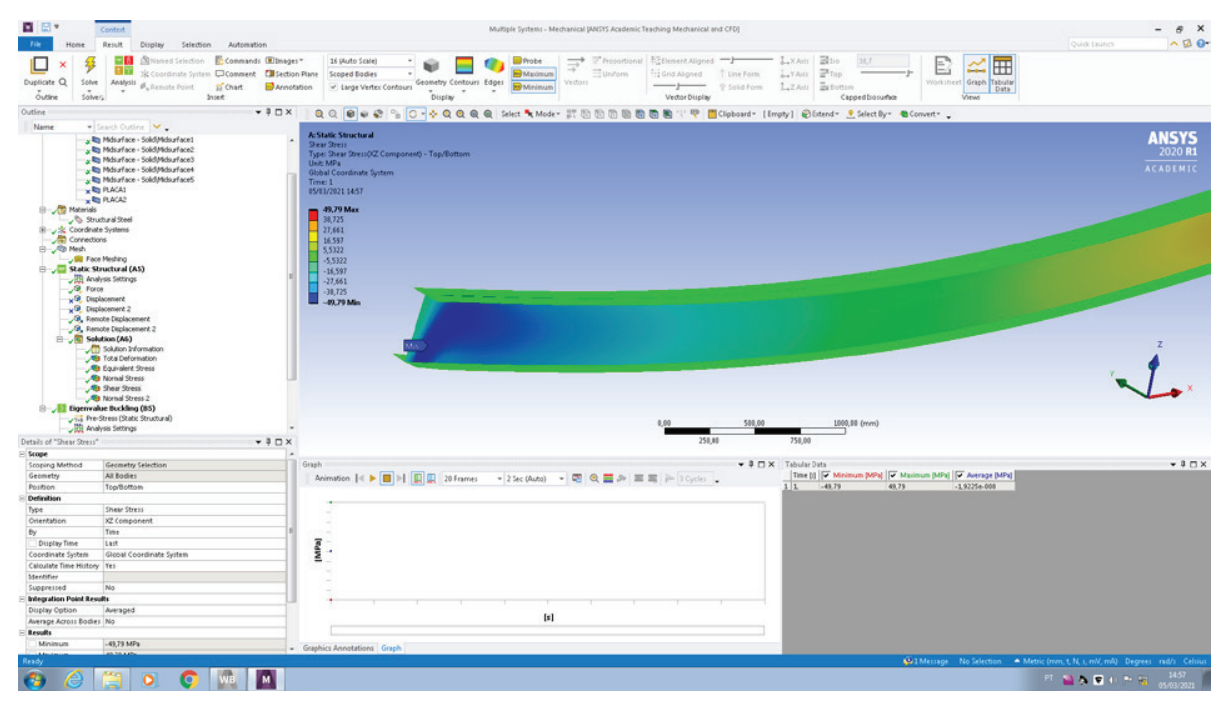

Figura 7 - Cisalhamento da viga. ().

Fonte: Autoria própria

\section{Resultados}

Os dados calculados analiticamente e aqueles obtidos pelo método de elementos finitos encontram-se ilustrados no quadro 1 :

Quadro 1 - Dimensão dos corpos de prova

\begin{tabular}{|c|c|c|}
\hline Objeto de análise & Valor analítico & Valor MEF \\
\hline Tensão normal $(\sigma)$ & $181,92 \mathrm{MPa}$ & $150,03 \mathrm{MPa}$ \\
\hline Tensão cisalhante ( & $37,94 \mathrm{MPa}$ & $49,78 \mathrm{MPa}$ \\
\hline Deflexão( & $27,69 \mathrm{~mm}$ & $24,71 \mathrm{~mm}$ \\
\hline
\end{tabular}

Fonte: Elaborado pelos próprios autores. 


\section{Utilização do ANSYS como laboratório virtual no ensino remoto}

Considerando os aspectos primários, verifica-se que é possível empregar a simulação numérica para observação do fenômeno e as diferenças entre a solução analítica e a experimental.

\section{Considerações finais}

O presente trabalho se propôs ilustrar a utilização do software ANSYS como um laboratório virtual. Partindo-se da premissa que a aprendizagem decorre de um processo de assimilação, aulas práticas têm por finalidade permitir ao aluno visualizar fenômenos descritos em sala de aula. No estudo de materiais deformáveis, como no caso de vigas biapoiadas, é possível realizar em laboratório ensaios de flexão que permitam comparar valores calculados analiticamente com os observados em ensaio. Utilizando o método de elementos finitos, verificou-se a possibilidade de realização da simulação computacional do comportamento de uma viga metálica, em substituição ao ensaio mecânico. A simulação serviria para visualização do fenômeno em ambiente virtual, de forma a refletir o comportamento mecânico no mundo real. Observou-se que a utilização do software ANSYS possibilita a realização destas simulações, com valores próximos ao analítico, algo que ocorreria de forma análoga ao ensaio mecânico. Portanto, guardadas as ressalvas e delimitações apresentadas no presente trabalho, conclui-se que é possível aplicar de forma satisfatória a simulação computacional com uso do ANSYS a título de laboratório virtual para ensaios mecânicos de flexão.

\section{Referências}

ASSOCIAÇÃO BRASILEIRA DE NORMAS TÉCNICAS. NBR 8800: Projetos de estruturas de aço e de estruturas mistas de aço e concreto de edifícios. Rio de Janeiro, p. 247. 2008. BRASIL. Ministério da Educação. RESOLUÇÃO Nº 2, DE 24 DE ABRIL DE 2019. <Disponível em: http://portal.mec.gov.br/>. Acesso em: 03 mar. 2021. 


\section{Utilização do ANSYS como laboratório virtual no ensino remoto}

CALLISTER. D., J. W. Ciência e Engenharia de Materiais - Uma Introdução. Grupo GEN, 2020. 9788521637325. Disponível em: <https://integrada.minhabiblioteca.com.br/\#/ books/9788521637325/>. Acesso em: 01 mar. 2021.

DEMO, Pedro. Educar pela pesquisa. 8. ed. Campinas: Autores Associados, 2007.

ENGENHRIA MECÂNICA UNIVERSIDADE TUIUTI. Bacharelado em Engenharia

Mecânica. Disponível em < https://utp.br/graduacao/bacharelado/engenharia-mecanica/>. Acesso em: 02 mar. 2021.

ESSS. Lab Virtual ESSS. Disponível em: < https://www.esss.co/biblioteca-tecnica/lab-virtualesss/>. Acesso em: 25 fev. 2021.

GUIA DE ENGENHARIA. Cálculo de flecha em viga pelo método da linha elástica. Disponível em: <https://www.guiadaengenharia.com/flecha-viga-linha-elastica/>. Acesso em: 05 mar. 2021

JACOB, F; TED, B. Um Primeiro Curso em Elementos Finitos. Grupo GEN, 2009. 978-85-216-1941-3. Disponível em: <https://integrada.minhabiblioteca.com.br/\#/ books/978-85-216-1941-3/>. Acesso em: 25 fev. 2021.

LAKATOS, Maria, L. E. Metodologia do Trabalho Científico. Grupo GEN, 2021. Disponível em: <https://integrada.minhabiblioteca.com.br/\#/books/9788597026559/>. Acesso em: 04 mar. 2021

RICHARD, B.; KEITH, N.J. Elementos de Máquinas de Shigley. Grupo A, 2016. 9788580555554. Disponível em: < https://integrada.minhabiblioteca.com.br/\#/ books/9788580555554/>. Acesso em: 26 fev. 2021

SOUZA, Sérgio Augusto. Ensaios Mecânicos de materiais metálicos. Fundamentos teóricos e práticos. São Paulo, Edgard Blucher, 1982.

TAFNER, Malcon MSc. A construção do conhecimento segundo Piaget. Revista Eletrônica de divulgação científica em neurociência. Disponível em: <http://www.cerebromente.org.br/ n08/mente/construtivismo/construtivismo.htm>. Acesso em: 02 mar. 2021 


\section{Utilização do ANSYS como laboratório virtual no ensino remoto}

UNIVERSIDADE TUIUTI. Comunicados Tuiuti COVID 19. Disponível em < https://www. tuiuti.edu.br/comunicados-tuiuti-covid19>. Acesso em: 03 mar. 2021.

Submetido em 07 de junho de 2021

Aceito em 16 de junho de 2021

Publicado em 8 de julho de 2021 